\title{
Interprofessional Intervention to Improve Geriatric Consultation Timing on an Acute Medical Service
}

\author{
Margaret Puelle, $M D, *$ Jocelyn Wiggins, $B M, B C h,{ }^{\dagger *}$ Rafina Khateeb, $M D, M B A,{ }^{\dagger \&}$

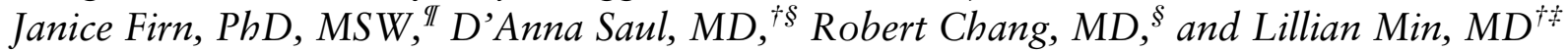

OBJECTIVES: To determine whether an interprofessional intervention would improve the use and timing of a geriatric consultation on a hospitalist service.

DESIGN: Difference-in-differences (DID), which measures the difference in improvement over time between intervention and control team patients attributable to the intervention.

SETTING: 1,000-bed U.S. academic medical center.

PARTICIPANTS: Individuals aged 60 and older admitted to a general medicine hospitalist service $(N=7,038 ; n=718$ on intervention teams, $\mathrm{n}=686$ historical controls, $\mathrm{n}=5,634$ on control teams (concurrent and historic).

INTERVENTION: On 2 of 11 hospitalist teams, a geriatrician attended multidisciplinary discharge rounds twice weekly and advised on the benefits of a geriatric consultation for individuals aged 60 and older.

MEASUREMENTS: Primary outcome was percentage of hospitalizations resulting in a geriatric consultation. Secondary outcome was days to geriatric consultation. Both outcomes were controlled for age, sex, comorbidity, mean daily intensity of inpatient care utilization, and admission in the prior 30 days. In the primary analysis, length of stay was controlled.

RESULTS: Intervention participants were more likely to have a geriatric consultation (DID $=2.35 \%$ absolute percentage points, $95 \%$ confidence interval $(\mathrm{CI})=0.59-4.39 \%$ ) and to have a consultation sooner (DID $=3.61$ fewer days, $95 \% \mathrm{CI}=-1$ to -7$)$.

From the *Department of Psychiatry, University of Pennsylvania, Philadelphia, Pennsylvania; ${ }^{\dagger}$ Division of Geriatric and Palliative Medicine, Department of Internal Medicine ISchool of Medicine, University of Michigan, Ann Arbor, Michigan; ${ }^{\circ}$ Center Geriatric Research, Education, and Clinical Center Ann Arbor Veterans Affairs Medical, Ann Arbor, Michigan; ${ }^{\circledR}$ Department of Internal Medicine, Division of Hospital Medicine School of Medicine, University of Michigan, Ann Arbor, Michigan; and the "Department of Learning Health Sciences School of Medicine, University of Michigan, Ann Arbor, Michigan.

Address Correspondence to Lillian Min, MD, MSHS, Associate Professor of Internal Medicine, University of Michigan Medical School Division of Geriatrics, 300 N. Ingalls Building, Ann Arbor, MI 48109.

E-mail: lmin@med.umich.edu.

DOI: 10.1111 /jgs. 15582

CONCLUSION: An interprofessional intervention that focused on hospitalist ordering practices increased use of appropriate geriatric consultation and decreased time to consultation. This model of interprofessional effort is effective. Future adaptations are needed to target scarce geriatric resources without increasing overall use. J Am Geriatr Soc 66:2372-2376, 2018.

Key words: geriatric consultation; discharge planning; quality improvement

$T$ he number of specialized geriatric providers has not kept pace with the increasing population of older adults, leading to calls for new models of care to increase delivery of geriatric expertise. ${ }^{1}$

Although units dedicated to the care of older adults have significant benefits, ${ }^{2-4}$ they are rare. A common alternative, geriatric consultation, has been associated with lower mortality and better functional status ${ }^{5,6}$ for selected clinical scenarios such as hip fracture ${ }^{7}$ and general traumatic injury, ${ }^{8}$ although a recent meta-analysis showed an effect only for short-term mortality. ${ }^{9}$

One reason that units may be more effective than consultation services is that units provide proactive geriatric evaluation, in contrast to consultation, which is reactive to clinical (e.g., delirium) and social (e.g., discharge disposition) complications. At our institution Michigan Medicine University of Michigan Medical Center, our motivation was to improve the timing and usefulness of multidisciplinary geriatric consultation for patients on the general medicine service. We desired to reduce consultations requested late in a patient's stay, sometimes on the day of potential discharge. With limited resources on our consultation team (1 each geriatrician, nurse, social worker), our aim was not to expand consultations to all older patients but to focus on those with greatest need. We hypothesized that our 
intervention would increase geriatric consultations and decrease the time to consultation, increasing proactive rather than reactive care. We used a difference-indifferences (DID) design, a stronger method than a simple pre-post comparison, to control for concurrent and potentially confounding changes in health care.

\section{METHODS}

\section{Setting and Design}

This study took place between July 1, 2013, and June 30, 2014, at Michigan Medicine, a 1,000-bed academic medical center, on the general direct care medicine hospitalist service, which consists of 11 teams each staffed by one physician hospitalist. Usual care includes weekday discharge rounds, a meeting attended by only by the hospitalist physician and case manager.

\section{Participant Sample and Data}

The original sample consisted of all admissions to the 11 hospitalist teams during the study period. The primary unit of analysis was each inpatient admission of an individual aged 60 and older. Individuals were considered to have received the intervention if they were admitted to an intervention team. We studied concurrent controls (teams not participating in the intervention) and historic controls (individuals hospitalized on all teams for 1 year before the intervention). The dataset was obtained from the administrative medical record and billing data and included information on sex, age, admission and discharge dates, death date, procedures and physician visits, diagnosis codes for all inpatient and outpatient care within 2 years, and diagnosis-related group.

\section{Intervention}

Two of the 11 teams agreed to participate in the intervention. No changes were made to the workflow of the remaining 9 teams. The intervention consisted of a geriatrician (1 of 5 rotating physicians) joining discharge rounds on Mondays and Thursdays-approximately 30 minutes for 2 team meetings. The geriatrician made case-by-case recommendations about which patients might benefit from a formal geriatric assessment. The attending hospitalist made the final decision regarding a formal consultation. A palliative care physician concurrently participated, with the focus on improving palliative care consultation orders and timing. (Results are reported elsewhere. $)^{10}$

\section{Measures}

\section{Exposure Variable}

Hospital billing data were used to match each day of hospital care to a hospitalist and then correlated with the weekly hospitalist schedule to determine team assignment. The modal physician was used to assign teams. If a patient changed teams during the hospitalization, the final team was assigned. For a small number of patients, no clear hospitalist team could be identified because an overnight physician admitted them, and they were discharged the next morning or transferred immediately to another service (e.g., intensive care, surgery). This method of determining team assignment was validated using blinded medical record review to determine the team providing care using a random sample of 200 patients, which yielded $96.8 \%$ agreement and kappa of 0.78 .

\section{Outcomes}

The primary outcome was change in proportion of admitted patients undergoing a geriatric consultation, measured as the absolute difference in percentage of hospitalizations with a consultation during the intervention year minus the percentage of hospitalizations with a consultation in the prior year. Thus, the hospitalization was considered to be the primary unit of analysis. We first matched the rotating geriatrician schedule to professional claims. Unbilled visits were not captured. Time to consultation was calculated as days from admission to date of first geriatric consultation note. This method of measuring the outcomes was validated using blinded chart review of a random sample of 200 hospitalizations (half with a known geriatric consultation), which yielded $98.2 \%$ agreement and kappa of 0.96 for occurrence of a geriatric consultation and agreement of $98.4 \%$ and kappa of 0.97 for the exact date of the initial geriatric consultation.

We controlled for factors affecting geriatric consultation: age, sex, prior recent admissions, Charlson Comorbidity Index (CCI), ${ }^{11}$ length of stay (LOS), and relative value units (RVUs) per day. We used a lookback period of 2 years for all inpatient and outpatient records to calculate the CCI. Recent readmission was defined as discharge within 30 days from the hospital system. RVUs per day were determined as an average of RVUs associated with all billed Common Procedural Terminology codes for procedures, evaluation, and management during the hospitalization.

\section{Analysis}

We first compared baseline characteristics of intervention and control patients using 1-way analysis of variance for continuous variables (age, RVUs/day, LOS, CCI) and chisquare tests for proportions (sex, recent admission). Our primary analysis included change in proportion receiving a geriatric consultation between the intervention and control groups over time using a DID model. This method ${ }^{12}$ compares pre- and postdata of the control and intervention groups to analyze differences in change of a proportion over time. The data are analyzed using logistic regression, with intervention versus control group as the primary predictor variable and the pre- versus post-time period indicator variable as a second predictor and the interaction between the two variables to evaluate the statistical significance of the DID. We used predicted probabilities resulting from the logistic regression model to determine the DID. ${ }^{12}$ We bootstrapped the sample 1,000 times to obtain $95 \%$ confidence intervals (CIs) (median, 97. $5^{\text {th }}$ and $2.5^{\text {th }}$ observations, holding continuous covariables at their means and categorical covariables at their modes). To control for clustering of patients within physicians who may have different practice patterns and responses to the intervention, we included a physician random effect. 
Second, we compared time to consultation of the intervention and control groups. We visually inspected KaplanMeier curves to ensure similar hazard functions and then used a Cox and then performed a Cox proportional hazard analysis. Then, we estimated the DID in days to consultation, determined by parametric survival analysis. This method is similar to determining median life expectancy, or the time over which half the population has died, but in this study, in which consultation occurred in many fewer than half of the patients, the few patients with long stays heavily influenced estimated median time to consultation, resulting in estimates of median time to -consultation that were longer than the typical LOS. Therefore, to test the DID associated with the intervention on a more generalizable hospital population, we based results on an earlier time-point, when $5 \%$ rather than $50 \%$ of the population has undergone a geriatric consultation. We controlled for the same factors as in the primary analysis except LOS (omitted because of correlation with exposure time). The baseline distribution of cumulative hazard was selected based on the lowest Akaike criterion. ${ }^{13}$ The final model was bootstrapped 1,000 times to obtain minimum expected days to achieve consultation on $5 \%$ or more of the population, using $97.5^{\text {th }}$ and $2.5^{\text {th }}$ estimates (units of whole days) as the $95 \%$ CIs, controlling for covariables held at their means/modes. We used Stata version 14 (Stata Corp., College Station, TX) for all analyses.

\section{RESULTS}

\section{Hospitalized Patient Characteristics}

Of 7,687 patients aged 60 and older admitted during the 2-year period, $7,038(91.6 \%)$ had sufficient data to assign to a hospitalist team (Supplemental Figure S1). There were no significant differences in patient age, sex, LOS, or recent admission between intervention and control hospitalizations (Table 1).

\section{Proportion Undergoing Geriatric Consultation}

\section{Univariate Analyses}

Of 7,038 hospitalizations analyzed, 307 (4.4\%) included a geriatric consultation. For teams receiving the intervention, the percentage including a geriatric consultation increased from $4.4 \%$ in the year before to $7.0 \%$ after the start of the intervention (a difference in absolute percentage points of $2.6 \%, 95 \% \mathrm{CI}=0.2-5.0 \%)$. Intervention teams also underwent more consultations during the intervention time than the concurrent control teams $(7.0 \%$ vs $3.7 \%$, a $3.3 \%, 95 \%$ $\mathrm{CI}=1.3-5.3 \%$ absolute percentage-point difference).

There was no significant secular change over time in geriatric consultation among the control teams $14.3 \%$ to $3.7 \%$, percentage-pt change over time $=-0.6 \%, 95 \% \mathrm{CI}=-$ $0.39-1.7 \%)$.

\section{Multivariable Analyses}

In the multivariable model, which adjusted for age, sex, recent admission, LOS, CCI, and controlled individual physician effects, the predicted percentage of patients undergoing a geriatric consultation increased by $2.2 \%$ absolute percentage points over the study period for intervention patients, compared to a $0.2 \%$ absolute percentage-point decrease in proportion of control patients undergoing consultations. This yielded a $2.3 \%$ absolute change in percentage points $(95 \% \mathrm{CI}=0.6-4.4 \%]$, the $\mathrm{DID}$, between the 2 groups (Figure 1). This increase in consultations would be an increase of 1.5 new consultations per week if this intervention were applied to the entire hospitalist service.

\section{Time to Geriatric Consultation}

The intervention group had the shortest time to consultation, as demonstrated by the cumulative proportion of patients undergoing a consultation (Figure 2). Using time to consultation as the outcome in a Cox proportional hazards model, the unadjusted hazard ratio (HR) associated with the DID was statistically significant $(\mathrm{HR}=1.87,95 \% \mathrm{CI}=1.10-3.16)$. Considering parametric survival models, the best-fit hazard distribution was log-normal. Controlling for covariables and holding covariables constant (at means or modes), expected time to undergo a geriatric consultation on $5 \%$ of admitted patients decreased by 2.1 days in the intervention group and increased by 1.5 days in the control group, yielding a net decrease, or mean DID, of 3.6 days (95\% CI=1-7 days).

\section{DISCUSSION}

New models to efficiently increase the effect of geriatricians are desperately needed. ${ }^{1}$ We found that shared interprofessional rounds between geriatricians and hospitalists

Table 1. Comparison of Patient and Hospitalization Characteristics Between Intervention and Control Team Patients During Intervention Phase $(\mathrm{N}=3,422)$

\begin{tabular}{llll}
\hline Characteristic & Control Group, $\mathbf{n = 2 , 7 0 4}$ & Intervention Group, $\mathbf{n = 7 1 8}$ & P-Value \\
\hline Age, mean \pm SD & $73.4 \pm 9.3$ & $73.6 \pm 9.3$ & .56 \\
Male, $n(\%)$ & $1,420(52.5)$ & $395(55.0)$ & .23 \\
RVUs per day, mean \pm SD ${ }^{1}$ & $7.8 \pm 3.9$ & $8.0 \pm 4.8$ & .34 \\
Charlson Comorbidity Index, mean \pm SD $_{\text {Readmitted patient, } \mathrm{n}(\%)^{2}}$ & $3.7 \pm 2.8$ & $3.7 \pm 2.8$ & .77 \\
Length of stay, days, mean \pm SD $^{2}$ & $485(17.9)$ & $116(16.2)$ & .26 \\
\hline
\end{tabular}

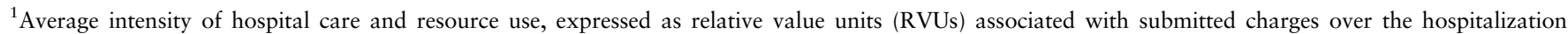
divided by length of stay.

${ }^{2}$ Admission date (for this hospitalization) was within 30 days of another inpatient discharge in this healthcare system.

$\mathrm{SD}=$ standard deviation.
} 


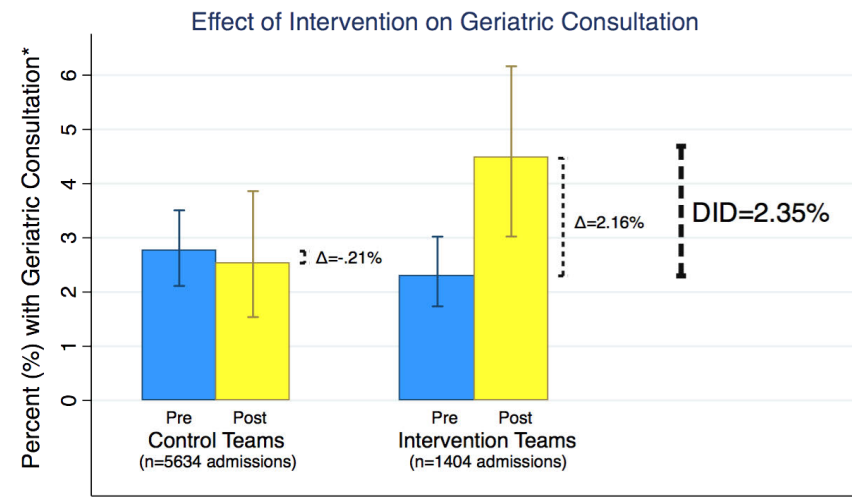

$\mathrm{DID}=$ Difference-in-Differences

*Adjusted for age, gender, co-morbidity, length of stay, readmission, care intensity; physician random-effect

Figure 1. Effect of intervention on geriatric consultation (difference-in-differences) $(\mathrm{N}=7,038)$. $*$ Predicted percentages are based on multivariable logistic regression model including full interaction between intervention and control and between postand pre-intervention time, random effect for physician, holding constant the mean or modal values of the covariables: a male patient aged 74, 7.8 average relative value units (RVUs) charged per day, Charlson Comorbidity Index of 3.7 points, length of stay of 6 days, with no 30-day recent hospitalization.

increased geriatric consultation by $2.3 \%$ of all older adults admitted to a general medicine hospitalist service and decreased time to consultation by nearly 4 days.

We increased the rigor of measure by comparing change over time for the intervention with that of a control group, using a DID approach, decreasing risk of biased results from secular changes in the healthcare system. Our findings suggest that a minimally resource-intensive model can effectively target complex services (geriatric consultation). Second, because we controlled for effect of individual physicians (who crossed intervention and control groups), our results suggest that the intervention was more important in delivering earlier geriatric consultations than hospitalist characteristics.

Our results should be viewed in light of several limitations. To allow for geriatric staff to participate, we limited the intervention to twice weekly. Therefore, patients who were admitted and discharged between enhanced discharge meetings could not directly receive the intervention. We included all patients in the analysis (intention to treat), which conservatively biased our findings toward no result. These results may not generalize to hospitals without capacity to handle additional geriatric consultations, although we believe that even hospitals with less-robust geriatric services have an interest in improving the efficiency of their specialty care. This intervention could be used to increase effectiveness of a lone geriatrician (e.g., to triage less-urgent geriatric questions to outpatient resources). A future direction would include detailed chart review for appropriateness and quality of care and a measure of how many unnecessary consultations were prevented (which we anecdotally noted but could not study in this design). Lastly, although we provided consultations earlier in the hospital course (fewer days to consultation), we could not test for effect on postconsultation length of stay. Such an investigation would require prospective recording of expected length of stay, which we plan for future study.

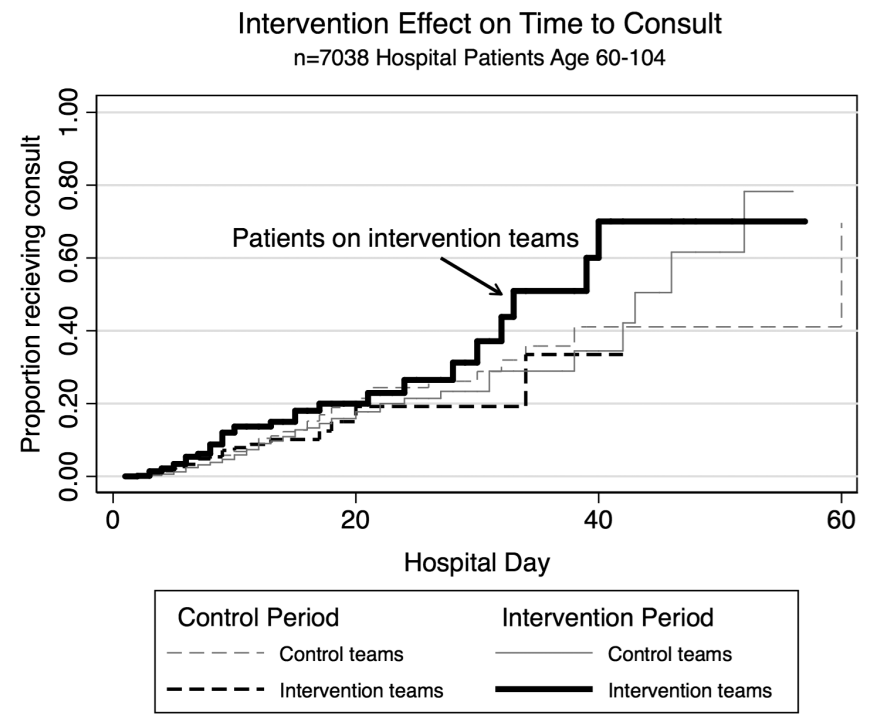

Figure 2. Intervention effect on time to geriatric consultation. Kaplan-Meier curves of cumulative proportion of sample receiving a geriatric consultation according to intervention versus control teams. The darker solid line represents the intervention teams during the intervention. Concurrent control teams are represented by the gray solid line, intervention team patients before the intervention by darker dashed line, and control team patients before the intervention by dashed grey line.

In conclusion, scheduled interprofessional rounds between hospitalists and geriatric specialists facilitate proactive person-centered use of scarce geriatric services.

\section{ACKNOWLEDGMENTS}

This research was presented, in part, at the 2016 Annual Scientific Research Meeting in Long Beach, California. This research was supported through Blue Cross Blue Shield of Michigan Foundation (BCBSM-15-PAF07639 01/2016-3/2017).

Conflict of Interest: The authors have no conflicts of interest to declare.

Authors Contributions: Puelle: data acquisition, analysis and interpretation of results, manuscript preparation, final approval. Wiggins: study design, data acquisition, final approval. Khateeb: study design, data acquisition, interpretation of results, manuscript preparation, final approval. Firn: study design, data acquisition, manuscript preparation, final approval. Saul: data acquisition, final approval. Chang: interpretation of results, final approval. Min: study design, data acquisition, analysis and interpretation of results, manuscript preparation, final approval.

Sponsor's Role: The funder of this work was Blue Cross Blue Shield of Michigan (PI Khateeb), which did not have any role in the interpretation of the results or manuscript preparation.

\section{REFERENCES}

1. Institute of Medicine. Retooling for an Aging America: Building the Health Care Workforce. Washington, DC; 2008.

2. Fox MT, Persaud M, Maimets I, et al. Effectiveness of acute geriatric unit care using acute care for elders components: A systematic review and metaanalysis. J Am Geriatr Soc 2012;60:2237-2245. 
3. Landefeld CS, Palmer RM, Kresevic DM, Fortinsky RH, Kowal J. A randomized trial of care in a hospital medical unit especially designed to improve the functional outcomes of acutely ill older patients. N Eng J Med 1995;332:1338-1344.

4. Counsell SR, Holder CM, Liebenauer LL, et al. Effects of a multicomponent intervention on functional outcomes and process of care in hospitalized older patients: A randomized controlled trial of Acute Care for Elders (ACE) in a community hospital. J Am Geriatr Soc 2000;48:1572-1581.

5. Hogan DB, Fox RA. A prospective controlled trial of a geriatric consultation team in an acute-care hospital. Age Ageing 1990;19:107-113.

6. Hogan DB, Fox RA, Badley BW, Mann OE. Effect of a geriatric consultation service on management of patients in an acute care hospital. Canad Med Assoc J 1987;136:713-717.

7. Marcantonio ER, Flacker JM, Wright RJ, Resnick NM. Reducing delirium after hip fracture: A randomized trial. J Am Geriatr Soc 2001;49:516-522.

8. Tillou A, Kelley-Quon L, Burruss S, et al. Long-term postinjury functional recovery: Outcomes of geriatric consultation. JAMA Surg 2014;149:83-89.

9. Deschodt M, Flamaing J, Haentjens P, Boonen S, Milisen K. Impact of geriatric consultation teams on clinical outcome in acute hospitals: A systematic review and meta-analysis. BMC Med 2013;11:48.

10. Khateeb R, Puelle MR, Firn J, Saul D, Chang R, Min L. Interprofessional rounds improve timing of appropriate palliative care consultation on a hospitalist service. Am J Med Qual 2018 Apr 1:106286061876806.

11. Deyo RA, Cherkin DC, Ciol MA. Adapting a clinical comorbidity index for use with ICD-9-CM administrative databases. J Clin Epidemiol 1992;45:613-619.

12. Karaca-Mandic P, Norton EC, Dowd B. Interaction terms in nonlinear models. Health Serv Res 2012;47(1 Pt 1):255-274.

13. Akaike H. A new look at the statistical model identification. IEEE Trans Autom Control 1974;19:716-723.

\section{SUPPORTING INFORMATION}

Additional Supporting Information may be found in the online version of this article.

Supplemental Figure S1: Flow of data (hospitalizations) from 2 years of administrative hospital data into four groups by time and intervention versus control in order to conduct a differences-in-differences study on change over time of outcomes.

Supplemental Figure S2: Our study design comes with an important limitation, namely that the trends in both groups must be parallel in the absence of the intervention (the counterfactual). We performed an additional analysis to examine the trends over time, focusing on the time prior to the intervention to estimate whether the trends would reasonably be parallel between the two groups in the absence of the intervention. We first arranged the proportion of consultations in the two groups by quarter of admission over the two years. The figure of this output is displayed below. There are gross seasonal variations in consultation activity throughout, but we believe that the trends prior to the intervention are reasonably parallel. 